\title{
Correction to: Pegvisomant in acromegaly: an update
}

\author{
A. Giustina ${ }^{1}$ - G. Arnaldi ${ }^{2}$ - F. Bogazzi ${ }^{3} \cdot$ S. Cannavò ${ }^{4} \cdot$ A. Colao $^{5} \cdot$ L. De Marinis $^{6}$. \\ E. De Menis ${ }^{7} \cdot$ E. Degli Uberti ${ }^{8} \cdot$ F. Giorgino ${ }^{9} \cdot$ S. Grottoli ${ }^{10}$. \\ A. G. Lania ${ }^{11} \cdot$ P. Maffei ${ }^{12} \cdot$ R. Pivonello ${ }^{13} \cdot$ E. Ghigo $^{14}$
}

Published online: 28 December 2017

(C) Italian Society of Endocrinology (SIE) 2017

\section{Correction to: J Endocrinol Invest (2017) 40:577-589 https://doi.org/10.1007/s40618-017-0614-1}

Unfortunately, in page 584, second column, the first sentence under the heading "Type of switch" has been published incorrectly. The complete correct sentence is given below.

In clinical studies, the primary goal of therapy is usually the achievement of a complete biochemical control.

The original article can be found online at https://doi. org/10.1007/s40618-017-0614-1.

A. Giustina

a.giustina@libero.it

1 Chair of Endocrinology, Vita-Salute San Raffaele University, Milano, Italy

2 Clinic of Endocrinology and Metabolism Disease, Ospedali Riuniti di Ancona, Ancona, Italy

3 Department of Clinical and Experimental Medicine, University of Pisa, Pisa, Italy

4 Department of Clinical and Experimental Medicine, University of Messina, Messina, Italy

5 Department of Clinical Medicine and Surgery, University of Naples Federico II, Naples, Italy

6 Pituitary Unit, Catholic University School of Medicine, Rome, Italy

7 Department of Internal Medicine, General Hospital, Montebelluna (TV), Italy

8 Section of Endocrinology and Internal Medicine, Department of Medical Sciences, University of Ferrara, Ferrara, Italy
9 Department of Emergency and Organ Transplantation, Section of Internal Medicine, Endocrinology, Andrology and Metabolic Diseases, University of Bari Aldo Moro, Bari, Italy

10 Endocrinology, Diabetology and Metabolism, AOU Città della Salute e della Scienza of Turin, Turin, Italy

11 Endocrinology Unit, Department of Biomedical Sciences, Humanitas University and Humanitas Research Hospital, Rozzano, Italy

12 Department of Medicine (DIMED), 3rd Medical Clinic, Azienda Ospedaliera Padova, Padova, Italy

13 Department of Clinical and Surgery Medicine, Endocrinology and Metabolism, University of Naples, Naples, Italy

14 Department of Medical Sciences, School of Medicine, University of Turin, Turin, Italy 